Itinéraires Itinéraires

Littérature, textes, cultures

\title{
Chanter par devoir de mémoire : une lecture postcoloniale de quelques textes du rappeur Médine
}

Singing as a Memory Duty: A Postcolonial Reading of Some Selected Songs of the Rap Musician Médine

\section{Julien Mowang Ngoula}

\section{OpenEdition \\ Journals}

Édition électronique

URL : https://journals.openedition.org/itineraires/8775

DOI : $10.4000 /$ itineraires.8775

ISSN : 2427-920X

Éditeur

Pléiade

\section{Référence électronique}

Julien Mowang Ngoula, «Chanter par devoir de mémoire : une lecture postcoloniale de quelques textes du rappeur Médine », Itinéraires [En ligne], 2020-3 | 2021, mis en ligne le 08 décembre 2021 , consulté le 12 décembre 2021. URL : http://journals.openedition.org/itineraires/8775 ; DOI : https:// doi.org/10.4000/itineraires.8775

Ce document a été généré automatiquement le 12 décembre 2021.

\section{(c) (†) $\odot$}

Itinéraires est mis à disposition selon les termes de la licence Creative Commons Attribution - Pas d'Utilisation Commerciale - Pas de Modification 4.0 International. 


\section{Chanter par devoir de mémoire : une lecture postcoloniale de quelques textes du rappeur Médine}

Singing as a Memory Duty: A Postcolonial Reading of Some Selected Songs of the Rap Musician Médine

Julien Mowang Ngoula

\section{Introduction}

L'histoire de l'Afrique est longtemps restée peu connue voir "falsifiée » lorsqu'elle devait être enseignée. Le philosophe allemand Hegel pense à ce propos que «L'Afrique n'est pas une partie historique du monde» ([1822] 1965). Cette assertion qui semble clairement nier l'existence d'une histoire africaine donnera lieu à de nombreux travaux dans le but d'interroger le passé du continent et faire connaître la vérité. Cheick Anta Diop (1967), Théophile Obenga (2000), Molefi Kete Asante (1998) et bien d'autres, vont consacrer l'essentiel de leurs productions à la restitution de la vérité historique, surtout celle faisant $\mathrm{du}$ continent noir le berceau de la civilisation. Allant vraisemblablement dans le sens déjà emprunté par les historiens suscités, Médine, rappeur franco-algérien, par "devoir de mémoire", entendu comme l'obligation morale de se souvenir d'un événement historique tragique et de ses victimes, afin de faire en sorte qu'un autre événement de ce genre ne se reproduise plus jamais, fait usage de la chanson et tente de rendre au monde en général et à l'Afrique en particulier certains épisodes de son histoire longtemps occultée par la pensée eurocentrique, qui fait de l'Europe la norme de jugement de l'histoire Universelle. À partir des titres «Alger pleure » (2012), « Kunta Kinté, enfant du destin » (2008), il revient sur les traces encore perceptibles de la guerre d'Algérie des années 1950, et sur la période esclavagiste dont a souffert le continent. À travers l'évocation de ce passé douloureux dont les séquelles restent encore perceptibles, le chanteur dévoile, en exorcisant le mal, toutes les vicissitudes du passé. Dans une perspective résolument postcoloniale telle 
que théorisée par Frantz Fanon avec le concept de conscience nationale, qu'il conçoit comme étant «la cristallisation coordonnée des aspirations les plus intimes d'un peuple " (Fanon 2002: 145), cette notion se positionne dès lors comme un catalyseur d'une prise de conscience de l'état d'urgence des ex-colonisés et de la nécessité de pondérer les forces endogènes dans le processus de reconstruction des états ayant subi l'esclavage et la domination occidentale. Notre réflexion interroge les motivations de l'engagement du rappeur et la manière dont ses textes constituent une (ré)écriture, mieux un lieu de la symbolique mémorielle de l'histoire oblitérée des figures de la résistance à ladite domination. En outre, nous postulons que Médine, dans un style teinté de lyrisme et d'humanisme, milite pour un ancrage du passé africain dans la mémoire collective, une prise de conscience par les Africains des défis existentiels actuels, en s'inspirant des voies empruntées par ceux qui ont payé de leurs vies pour leur liberté.

\section{De l'évocation de l'histoire à son ancrage dans la mémoire collective}

2 Le devoir de mémoire s'appréhende comme : l'exhortation à ne pas oublier les crimes du passé afin que ces derniers ne puissent se rejouer dans nos sociétés contemporaines (Kattan 2002 : 147). Ce concept fut employé dans les années 1990 pour caractériser les atrocités commises lors de la tragédie de la Shoah. En effet, après la seconde guerre mondiale, les rescapés des camps de concentration et d'extermination décident de pérenniser le souvenir de leurs camarades disparus, victimes de la barbarie nazie. Par la suite, cette volonté de combattre l'oubli sera reprise dans l'espace public pour devenir par l'entremise des survivants ce que l'on appellera communément le devoir de mémoire (ibid. : 147).

3 La mise en récit de l'histoire n'a pas toujours fait l'unanimité chez les responsables des pratiques inhumaines administrées soit à une population, soit à une race, jugées inférieures contre lesquelles des barrières de tous genres ont été érigées, empêchant de fait son élévation, son épanouissement. Il va de soi que la falsification du passé de l'Afrique par les adulateurs de la colonisation, qui trouvaient en l'expansion coloniale européenne « une mission civilisatrice » des populations non européennes, considérées comme primitives, voire "sauvages", n'est pas un fait anodin dans la mesure où le projet était d'« européaniser » le reste du monde. Lescarbot pense en effet que François $\mathrm{I}^{\mathrm{er}}$ était « désireux d'accroitre le nom Chrétien et François» (Lescarbot 1795). Selon la position occupée dans le déroulement des événements, l'on peut être porté, soit à la propagation des faits, soit à leur embrigadement, surtout quand on a été du côté oppresseur.

4 Après certains événements, certaines victimes, refoulent toute idée de revenir sur ce qu'elles ont vécu et qu'elles jugent psychologiquement lourd à supporter. C'est le cas de la Shoah, du génocide rwandais, des bombardements de Nagasaki et de Hiroshima, dont la seule évocation peut susciter encore de la douleur chez les victimes et leurs descendants. Il y a lieu de dire qu'aussi longtemps que des atrocités seront commises, il y aura une propension à les dissimuler, peut-être par culpabilité, ou du fait du traumatisme qu'on a subi, et qu'on voudrait évacuer. Face à la difficulté persistante des deux parties à dévoiler les histoires vécues, l'artiste se donne pour mission, dans une 
perspective de restitution de la mémoire enfouie, de ressusciter le passé enseveli, afin de l'actualiser et le faire connaître de tous, surtout à des fins de réparation.

\section{« Kunta Kinté » ou les empreintes de la période esclavagiste}

5 Médine fait une exploration du passé agité, pas seulement de l'Afrique mais également d'autres sociétés, dont le parcours historique mérite un temps d'arrêt pour le transmettre aux générations présentes et futures. Puisant son inspiration dans l'histoire de l'esclavage en Afrique de l'Ouest, il fait la projection d'une société ancrée dans son passé, dispositif à partir duquel celle-ci visualise son futur et vit mieux son présent. Cette symbolique de l'écriture de l'histoire est perceptible dans le contenu du titre « Kunta Kinté, enfant du destin ».

6 Le personnage Kunta Kinté dont le nom est d'origine Mandingue s'assimile à un mythe, à une légende, du fait des incertitudes qui planent sur son existence. Présenté comme un personnage de fiction et héros du roman Roots d'Alex Haley (1976), Kunta est érigé en emblème de la résistance contre l'esclavage.

7 Bien qu'ayant été assez controversées, les sources orales qui ont conduit à la publication du roman situent les origines de Kunta en Gambie, précisément dans la tribu Mandingue. Fils de Binta et d'Omoro Kinté ${ }^{1}$, ce n'est pas nécessairement la bataille autour de son existence, encore moins de ses origines qui semble attirer l'attention et être la source de motivation de tous ces artistes qui ont produit des chansons en hommage à celui qui, fier de ses origines africaines et de son identité, s'est opposé systématiquement à l'esclavage. Les valeurs qu'il incarne et surtout celles véhiculées par ses actions d'opposant à l'esclavage sont de toute évidence autant de raisons qui expliquent l'obsession de certains artistes à l'évoquer dans leurs textes.

Un regard panoramique sur le rap de ces deux dernières décennies montre que ce personnage représente un héritage pour les rappeurs aussi bien français qu'américains, qui, à travers lui inscrivent l'esclavage dans la mémoire collective par une évocation constante de ses luttes. Kunta Kinté devient donc la figure de cette Amérique qui se souvient de ses origines africaines. Que ce soit Kendrick Lamar dans son titre « Kunta Kinté », Busta Rhymes dans « Rhymes Galore », ou encore la rappeuse Missy Elliott avec le titre "Work it » (2012), il y a lieu de dire que le personnage de Kunta Kinte est une légende dans la sphère du hip-hop. En France comme en Amérique, sa figure est assimilée à l'esclavage, au racisme anti-Noirs, à la colonisation, et aux souffrances subies par les populations noires à cette époque. Du rappeur IAM dans «Tempérament Kunta Kinté » 2017, à Leck «All in » 2013, en passant par Sefyu «La légende » 2006, Kunta symbolise les souffrances connues par les Noirs depuis l'esclavage comme on peut le lire dans ces propos Sefyu :

demande à Sami Naceri si la première fois

qu'un arabe échappe au contrôle de routine

c'était pas dans un taxi

Complexés dans tous les scénarios les Noirs meurent les premiers

Ça passe bien depuis Kunta Kinté ${ }^{2}$

Contrairement aux artistes qui se limitent à l'évoquer sans scruter en profondeur son passé, ses origines, Médine lui consacre toute une chanson. En 2008, dans son titre «Kunta Kinté, enfant du destin », il donne une vibrante poésie en hommage à cette 
figure de la résistance. L'artiste revient sur son quotidien depuis le petit village de Djouffouré jusqu'à sa déportation comme esclave.

\section{«Alger pleure » : aux sources des événements d'Algérie}

10 La guerre d'Algérie reste incontestablement l'une des luttes de libération ayant marqué l'Algérie et la France, ainsi que le monde puisque plusieurs autres pays à l'instar de l'URSS, des USA, de l'Égypte, de l'Allemagne de l'Est, de Cuba et la Corée du Nord, s'y retrouveront mêlés en apportant un appui militaire, diplomatique, en médicament, en armement à l'Algérie. La France quant à elle sera aidée principalement par l'OTAN qui lui apportera un soutien logistique, diplomatique et en armement. La guerre se déroule de 1954 à 1962 en terre algérienne, alors colonie française depuis 1830. Elle s'achève par l'octroi de l'indépendance à l'Algérie par la France le 5 juillet 1962. Pour ce qui est des pertes, de centaines de milliers de morts qui se comptabilisent aussi bien dans les rangs de l'armée française que du côté des nationalistes algériens. Appelée «la guerre de libération » ou "événements d'Algérie » en son temps, elle opposa l'armée française aux insurgés algériens, dont le combat consistait à défendre la souveraineté algérienne. Face à l'ampleur du conflit, «plus d'un million de colons européens ont été contraints de fuir le pays » (Office of the United Nations High Commissionner for refugees 2000). Le fait que Médine y consacre un texte témoigne du contexte tumultueux que cette période a joué et joue dans la politique interne algérienne et des relations que le pays entretient avec la France. L'on pourrait comprendre le choix du rappeur de marquer un temps d'arrêt sur cette période du fait déjà de ses origines algériennes d'une part et de sa nationalité française d'autre part.

\section{Médine : historien du passé et poète de la mémoire collective}

11 Le processus de restitution du passé aux générations présentes et futures par Médine à travers ses choix thématiques s'abreuve aux sources des périodes esclavagistes et coloniales. En effet, les textes sont le lieu pour l'artiste de revenir sur certains maux générés par la rencontre entre l'Afrique et l'Occident depuis le grand commerce triangulaire jusqu'à la colonisation dont l'Afrique souffre encore. Cette position rejoint celle d'Aimé Césaire qui affirme que :

le grand drame historique de l'Afrique a moins été sa mise en contact trop tardive avec le reste du monde, que la manière dont ce contact a été opéré ; que c'est au moment où l'Europe est tombée entre les mains des financiers et des capitaines d'industrie les plus dénués de scrupules que l'Europe s'est "propagée »; que notre malchance a voulu que ce soit cette Europe-là que nous ayons rencontrée sur notre route et que l'Europe est comptable devant la communauté humaine du plus haut tas de cadavres de l'histoire. (Césaire $1955: 14$ )

Malgré ce doigt accusateur que pointe le poète sur l'occident, il y a lieu de comprendre que, loin de condamner avec véhémence la rencontre entre l'Afrique et l'Europe, ces artistes s'indignent des méthodes employées par l'Occident pour établir le contact avec l'Afrique.

12 À partir de la thématique développée dans ses chansons, Médine, connu pour son style engagé, captive surtout l'auditeur par son timbre vocal qui traduit son ressenti face aux événements décrits. Si dans le texte "Alger pleure » l'on retrouve un rythme plutôt 
lent, fluide et une mélodie assez douce, « Kunta Kinté » est une alternance des rythmes lent et rapide en fonction des passages. Soucieux de la beauté du message délivré, le rappeur choisit la rime plate pour les contours esthétiques des textes :

J'écarte la végétation qui me gêne

Mes pieds écorchés piétinent ma terre gambienne

Quand bien même je continue ma course

Poursuivi depuis l'aube par je ne sais quelle bête de la brousse (« Kunta Kinté »,

v. 1-4)

J'ai l'sang mêlé : un peu colon, un peu colonisé

Un peu colombe sombre ou corbeau décolorisé

Médine est métissé : Algérien-Français

Double identité : je suis un schizophrène de l'humanité

De vieux ennemis cohabitent dans mon code génétique

À moi seul j'incarne une histoire sans générique («Alger pleure », v. 40-45)

Par ces procédés généralement propres au rap, il témoigne et fait revivre, tout en les dénonçant, les atrocités de ces différentes périodes pendant lesquelles pour sa couleur de peau, le Noir était soumis à toute sorte de maltraitances. Bien plus, il dresse un bilan qui témoigne des séquelles encore présentes sur les populations victimes des exactions des bourreaux. La violence, la résistance, la liberté, la question identitaire, etc., sont, entre autres, les différentes thématiques abordées par l'auteur pour exprimer sa pensée.

\section{De la violence comme expression du malaise africain}

À la lecture des textes " Kunta Kinté » et "Alger pleure ", on remarque que la violence sur les Africains était une des méthodes privilégiées par les colons pour mettre en œuvre leurs desseins, celui de déporter les Noirs vers les plantations d'une part, et de les assimiler à la culture occidentale, d'autre part. Le récit des événements liés à la capture de Kunta et le traitement administré aux Algériens qui s'opposaient à la France sont une parfaite illustration de la barbarie humaine. Ce point est d'autant saillant que dans le village de Djouffouré où naquit Kunta, la seule évocation du nom Toubab ${ }^{3}$ provoquait une sorte de panique, une peur chez les populations. Cette situation fut également vécue par les Algériens à l'évocation du général Aussarress ${ }^{4}$. Médine le dit dans ce passage :

à djouffouré, village de l'Afrique occidentale,

[...]

Où tout bas, on évoque les toubabs

Pour effrayer les enfants, le soir, sous un baobab (« Kunta Kinté », v. 22-27)

Alors que Kunta se trouve en brousse comme à l'accoutumée pour une partie de chasse, il sera vite frappé par un sentiment inhabituel : subitement, son compagnon de chasse,

... le chien n'aboie plus

[...] c'est le toubab et ses alliés (« Kunta Kinté », v. 60-61)

Un objet lourd lui écrase l'épaule,

$[\ldots]$

Un coup de fouet réduit ses vêtements en pièces

Un deuxième lui dépèce l'épiderme des fesses (« Kunta Kinté », v. 62-67)

D'un texte à l'autre, le constat est le même: l'artiste revient sur les tortures administrées aux populations «indigènes » et «non civilisées » pendant la guerre d'Algérie. En effet, le colon ne lésinait sur aucun moyen pour parvenir à ses fins. Et à Médine de rentrer dans un jeu d'interrogations rhétoriques, aux enjeux ô combien 
importants et interpellateurs, afin de se remémorer tous ces moments qui attestent d'un trauma refoulé qui fait retour dans l'imaginaire psychologique des victimes, comme l'artiste l'indique :

Pensiez-vous qu'on oublierait la torture?

Que la vraie nature de l'invasion était hydrocarbure?

Pensaient-ils vraiment que le pétrole était dans nos abdomens,

Pour labourer nos corps comme on laboure un vaste domaine? («Alger pleure»,

v. 48-51)

De ces différentes questions qui s'adressent également aux ex-colonisateurs, il semble vouloir attirer l'attention sur le mal qui a été commis et le traumatisme qui habite certaines victimes et leurs descendants. Il ne s'arrête pas là ; car il rappelle toutes les tortures que subissaient les forçats et les opposants à l'entreprise coloniale. Le manque d'humanisme de leurs bourreaux se traduit chez lui par l'évocation des conditions de déportation des esclaves vers leur nouvelle destination, lieu où ils seront contraints à s'accommoder à un mode de vie sans précédent ; celui imposé par leur statut de captifs.

Le négrier qui les transporte est présenté comme une grosse bête qui avale ses occupants, mieux, est comparé à une geôle. Il s'agit d'un espace carcéral où l'humanisme n'a pas droit de cité, où la dignité humaine est complètement bafouée. Après cette longue bataille qui a tout de même abouti à la capture de Kunta, il revit son rêve :

... s'éveille

Dans le ventre de la bête qu'il n'avait vu qu'en sommeil

Le corps gisant dans ses propres déjections

Infection d'une centaine de corps en ébullition

Matière fécale et vomissures purulentes blessures brûlantes

Sur fond de mort pullulante, fulgurante douleur entre les omoplates ( Kunta

Kinté ", v. 82-87)

Les traitements subis par les esclaves et décrits par l'artiste font écho avec ce que les Algériens ont vécu pendant la guerre d'indépendance de leur pays, puisque le même traitement était administré par les colons pour assimiler et continuer à exercer la suprématie politique française en Algérie. La résistance des Algériens déclenchera le conflit dont les pertes en vies humaines sont estimées entre 140000 et 152863 combattants du côté du FLN, qui réclamait par ses actions « La restauration d'un État Algérien souverain, démocratique et social dans le cadre des principes islamiques " (Delaveau 1989: 64). De l'autre côté, on comptabilisera 25600 soldats français tombés. Si les chiffres sont assez considérables pour l'ensemble des combattants, ils le sont encore plus du côté des civils. Les Algériens morts sont estimés à plus de 250000 et les déplacés à plus de 3000000 . Quitter son pays était un choix imposé par la situation de guerre qui prévalait. Toutefois s'il fallait faire le choix de partir,

On n'voulait pas d'une séparation de crise

De n'pouvoir choisir qu'entre un cercueil ou une valise (« Alger pleure », v. 13-14)

Au regard de bien d'autres guerres de conquête qu'a connues le monde, aucun projet d'occupation ne peut véritablement se faire sans opposition, puisque l'instinct de survie qui caractérise l'homme l'oblige à faire obstacle, chaque fois qu'il s'estime être menacé. Face à ce refus de la part des Algériens de se voir contraindre à de nouvelles obligations sur leur propre terre, le colon, déterminé à imposer sa pensée, va user de méthodes dénuées d'humanisme. L'auteur évoque celles-ci et les exprime dans un lyrisme qui trahit son malaise, sa gêne face aux souffrances occasionnées par l'idée de 
conquête d'une entreprise dominatrice aux ambitions démesurées. Les vers suivants sont une manifestation de ce ressenti chez l'artiste, et qu'il extériorise en ces termes :

On ne peut oublier le code pour indigènes

On ne peut masquer sa gêne, au courant de la gégène

Électrocuter des hommes durant six ou sept heures

Des corps nus sur un sommier de fer branché sur le secteur

On n'oublie pas les djellabas de sang immaculées

La dignité masculine ôtée d'un homme émasculé

Les corvées de bois, creuser sa tombe avant d'y prendre emploi

On n'oublie pas les mutilés à plus de trente endroits

Les adverses de coup

Le supplice de la goutte

Les marques des bottes sur l'honneur des djounouds

On n'oublie pas les morsures du peloton cynophile

Et les sexes non circoncis dans les ventres de nos filles

On n'omet pas les lois par l'omerta (« Alger pleure », v. 52-65)

La violence et le manque d'humanisme chez les bourreaux sont également perceptibles dans le traitement qu'ils administrent à la femme noire. Dans une posture qui trahit son caractère à la fois humaniste et féministe, le poète s'interroge et semble être dans une quête permanente de compréhension de l'état d'esprit des «Toubabs » qui, visiblement n'ont aucune crainte, et pas de remords face aux actes dont ils sont coupables. Médine s'interroge dès lors sur cette curiosité en ces termes :

Les toubabs n'ont-ils pas de divinité ?

N'ont-ils pas d'enfants ou d'épouses à aimer?

Puisqu'ils violent, tuent et souillent de leur sperme

La virginité de nos princesses africaines

Ces toubabs n'ont-ils pas de dignité ?

N'ont-ils pas de savoir-vivre à enseigner?

Puisqu'ils frappent, fouettent et bien pire encore

En laissant pour morts les moindres recoins de nos corps (« Kunta Kinté », v. 92-99)

Cette perception de la femme africaine par son bourreau comme un objet de satisfaction de sa libido contraste avec l'image que les écrivains africains lui donnent. Dans « Femme nue, femme noire » (Senghor 1956), le poète rend un vibrant hommage à la femme, mère de l'humanité. Cette célébration atteint son paroxysme quand la femme est glorifiée et considérée par Grâce Etonde Ekotto comme «la première merveille du monde » (Ekoto $1987: 47$ ). Il convient de repréciser que la mise sur pied des entreprises esclavagistes et coloniales n'a pas prospéré sans résistance des populations opprimées. Qu'il s'agisse des esclaves ou encore des colonisés, chacun a, à travers des figures comme Kunta ou les insurgés algériens, marqué son opposition à la domination occidentale.

\section{Les manifestations de la résistance}

Le constat fait état que d'un conflit à l'autre, les méthodes employées par les opposants ne sont pas forcément identiques malgré l'objectif commun de libérer son peuple. Nous concevons la résistance comme le refus d'obéir, de céder à un système violent, injuste et de domination. Cette conception correspond à la posture qu'adoptent Kunta d'une part, et la résistance algérienne d'autre part.

Le quotidien des populations de Djouffouré en général, celui de Kunta en particulier, connaîtra un bouleversement, conséquence des différents enlèvements dont sont 
victimes leurs braves fils. Ils seront enlevés avec la complicité de leurs frères pour être emportés vers un Nouveau Monde, où ils seront forcés à travailler dans des plantations de canne à sucre et de coton. Toutefois, ceux-ci, à l'image de Kunta, ne se laisseront pas capturer sans résistance. Leur opposition témoigne de leur refus d'adhérer à la volonté des bourreaux. S'il est vrai qu'« on ne reçoit un étranger que de la manière dont il $v_{\text {vient }}^{5}$ ", il va de soi que les esclaves, les peuples colonisés, bien qu'ayant été victimes de toute sorte de maltraitances, ne pouvaient qu'opposer une fin de non-recevoir aux Occidentaux dont la venue en terre africaine était motivée par les idées de déportation, de colonisation, d'exploitation et d'assimilation. En effet, dans cette partie de chasse dont la cible est Kunta, il va, suite à sa résistance, être poussé à la soumission par de coups de fouets qui lui sont administrés. Cependant, il est fidèle à sa tradition et téméraire dans sa pensée :

Il déteste que des noirs lâches aident à la débauche

Que sa personne soit l'objet des négoces

Et que les traîtres soient peut-être de sa caste

Des noirs qui, contre un miroir, leur Histoire saccagent

Des chasseurs au service d'un armateur

Qui deviendront sûrement la proie de leurs employeurs

Une fois le travail accompli, les complices

Redeviendront de la marchandise ("Kunta Kinté », v. 70-77)

Face à cette trahison, Kunta résiste et use de ses techniques de lutteur pour rendre sa capture plus pénible :

Il dépêche au passage une pierre qu'il décoche

À toute force dans le visage le plus proche (« Kunta Kinté », v. 68-69)

Son opposition ne s'arrête pas là, puisqu'il va instiguer le mouvement de révolte que connaîtra le négrier qui les transporte. Tous les moyens sont mis à contribution pour manifester leur opposition à leur déportation ; il use de la langue maternelle :

Le mot mutinerie en Mandingue est prononcé

Et de sa chaîne émoussée, il étrangle le geôlier

Bascule à bâbord c'est le fouetteur qu'il bouscule

À tribord libère ses compagnons de cellule

Bientôt le ponton sera rempli de foyers

D'assez de guerriers pour dérouter le voilier (« Kunta Kinté », v. 105-110)

La témérité qui les caractérise est à l'image de la résistance que le FLN et l'ALN opposent à la France; elle dont l'ambition est de coloniser par tous les moyens l'Algérie, faisant fi de ses droits et libertés. En effet, ayant pris conscience de la détermination des groupes de libération de l'Algérie, le général de Gaulle à Alger en juin 1958 prononce un discours dans lequel il «lance un appel à la réconciliation». Mais le FLN va répondre par une intensification des luttes. Une fois de plus le président français va offrir une "paix des braves " qui sera rejetée en octobre 1958. Face à cet autre refus, de Gaulle déclare aux militaires français que, « le problème algérien ne sera réglé qu'après la victoire des armes françaises » (Delaveau et al. $1983: 65$ ).

\section{De la résistance à la quête d'une liberté}

21 La liberté n'ayant pas de prix, elle s'avère également être l'une des plus grandes aspirations des peuples. C'est d'ailleurs l'une des raisons qui justifient que, chaque fois qu'une société voit sa liberté bafouée, elle la défend et est prête à faire usage de tous les moyens pour atteindre son objectif, même au prix du sang. Connues pour être une autre forme de reconnaissance de la dignité humaine, les batailles pour les libertés ont 
marqué l'histoire du monde et à chaque fois, les raisons qui motivent ces luttes ont presque toujours été les mêmes. Médine en donne quelques-unes, qui manifestement témoignent de ce refus de soumission à une force qui bouleverserait les habitudes quotidiennes en établissant d'autres règles de fonctionnement dont les conséquences seraient perceptibles, sur le plan politique mais aussi socioculturel, comme on peut le lire à travers ces vers :

On n'voulait pas d'une séparation de crise

De n'pouvoir choisir qu'entre un cercueil ou une valise

Nous ne voulions pas non plus d'une Algérie Française

Ni d'une France qui noie ses indigènes dans le fleuve de la Seine («Alger pleure »,

v. 13-16)

Outre ces raisons suscitées et évoquées par les peuples hostiles à la domination occidentale, il y a lieu de préciser qu'une autre raison, et non des moindres, qui soustend cette opposition a été la crainte que ce conflit culturel s'ouvre sur une crise identitaire.

\section{Colonisation : du métissage à la crise identitaire}

L'identité est un terme polysémique. Elle a une conception spécifique qui dépend de son usage. Dans le contexte de cet article, ses définitions s'appliquent en sciences sociales et humaines. En sociologie, elle s'appréhende comme la reconnaissance de ce qu'est un individu, par lui-même et par ses alter ego. Cette acception s'ancre dans un double dynamisme : elle met d'abord en exergue, la reconnaissance d'une existence en tant qu'être individuel et singulier. Ensuite, elle envisage une reconnaissance d'une appartenance à un groupe social avec lequel l'Homme a en partage des valeurs socioculturelles et identitaires auxquelles le groupe s'identifie. Ce concept de crise identitaire est corollaire à l'état identitaire, culturel et social spécifique algérien, lui aussi consécutif aux multiples bouleversements politiques et socioculturels, lesquels ont continuellement marqué l'histoire de l'Afrique. Les réalités sociopolitiques et culturelles de l'environnement assez morose servent de vaste creuset à l'entreprise créatrice artistique de Médine. Il s'identifie à travers l'usage de la première personne du singulier, comme une victime de toutes les atrocités et métissages qu'a connus l'Algérie, et il déclare :

J'ai l'sang mêlé : un peu colon, un peu colonisé

Un peu colombe sombre ou corbeau décolonisé

Médine est métissé : Algérien-Français

Double identité : je suis un schizophrène de l'humanité

De vieux ennemis cohabitent dans mon code génétique

À moi seul j'incarne une histoire sans générique («Alger pleure », v. 1-6)

Médine, en réaction à l'état déboussolé des "identités carbonisées ${ }^{6}$ » (" Alger pleure ») et à la crise de la mémoire au sein de sa société, insère l'histoire dans ses textes. Dans une optique moins revancharde que didactique, la diffusion et l'enseignement du contexte sociohistorique et politique algérien sont un appel à l'acceptation et à la réconciliation des origines aux générations contemporaines. Il s'agit là d'une entreprise de dévoilement en vue de relever l'histoire et la mémoire de ce peuple encore meurtri par ce passé flou qui accentue l'hybridité des populations. 


\section{Médine : entre restitution de l'histoire africaine et construction prospective} vraisemblablement la mal gouvernance qui la caractérise tant sur le plan politique, économique, que socioculturel. Malheureusement cette situation ne favorise pas son éclosion, mais participe à son enfermement. Ses fils semblent alors atteints d'une schizophrénie culturelle dont les plus grandes manifestations sont au quotidien, l'acculturation, le déni de soi, la glorification de l'ailleurs. Conscient du fait que ces attitudes ne peuvent que continuer à faire obstacle au développement psychosomatique de l'Africain, Médine se fait lanceur d'alerte, mieux l'éveilleur de consciences.

\section{De l'encrage mémoriel à l'éveil de consciences}

Les titres « Kunta Kinté, enfant du destin » et «Alger pleure » s'inscrivent dans une dynamique rétrospective de l'histoire de la période esclavagiste qu'a connue l'Afrique, et celle coloniale algérienne, afin d'en tirer les éléments nécessaires à une " construction prospective ${ }^{7}$ » de la société. La démarche artistique du rétrospectif mémoriel est hautement significative pour la pérennité d'une civilisation. En effet, par sa diffusion et son appréhension, elle stimule chez le destinataire l'émergence d'une volonté d'ancrage de la mémoire historique et culturelle de sa communauté dans sa propre conscience. Le style de Médine s'articule autour de la reconnaissance des faits marquants de l'histoire ayant favorisé la genèse et la posture identitaire contemporaine des Africains.

En outre, sur un tout autre plan, l'auteur voudrait combler l'amnésie inhérente au passé africain longtemps oblitéré, et à l'identité algérienne aujourd'hui opaque, en inscrivant l'histoire du continent dans la conscience collective universelle. Il affiche clairement une volonté de transgression de ce qui pourrait s'assimiler à la loi de l'omerta, qui préconise le silence, même quand la situation est jugée grave. Chanter à des fins uniquement esthétiques devient dès lors proscrit chez Médine. Il rentre ainsi en accord avec la conception sartrienne de l'œuvre d'art, pour qui : «L'écrivain engagé sait que la parole est action; il sait que dévoiler c'est changer et qu'on ne peut dévoiler qu'en projetant de changer. Il a abandonné le rêve impossible de faire une peinture impartiale de la société, de la condition humaine » (Sartre 1948 : 125).

Dans cet ordre d'idées, il se propose de répondre aux besoins du peuple. Il dénonce les actes d'impérialisme qui sont coupables de la situation alarmante du continent, les maux et les fléaux qui gangrènent son environnement sociopolitique, culturel et identitaire tout en disposant dans son imaginaire, des signes et symboles qui proposent des solutions à même d'assainir ce climat qui est encore très loin d'être serein. Il se revêt d'une mission : celle de déconstruire le passé ficelé par les bourreaux d'hier, de faire connaître la vraie histoire, de guérir son peuple des tares sociohistoriques, psychologiques, identitaires, des schèmes mentaux enfouies dans leur mémoire profonde, et malheureusement érigés en vérités historiques auxquels ils sont rattachés. Un des bilans qui ne fait presque jamais l'unanimité c'est celui concernant le nombre de victimes : 
En février 1943, le nationaliste Ferhat Abbâs met au point «Le Manifeste du peuple algérien », qu'il complète par un « Projet de réformes » faisant suite au Manifeste. Il réclame la constitution d'un État algérien, autonome et démocratique, lié à la France par des relations de caractère fédéral. Le Projet sera rejeté. (Delaveau et al. $1983: 61)$

Ces différents rejets ouvriront sur des contestations à l'instar de celle du 8 mai 1945 qui se solde par des émeutes sanglantes, avec une centaine de Français massacrés. Dans une riposte farouche, les forces armées rentrent en conflit avec les nationalistes mélangés au peuple qui exige son autonomie. Les chiffres en termes de pertes sont estimés à 1500 morts par l'administration coloniale, ce qui est vivement contesté par les nationalistes qui parlent de 50000 morts. Contrairement au fait que ces nationalistes soient considérés comme des extrémistes, qualification répandue dans l'opinion, Médine se souvient et présente le Front de libération nationale comme un mouvement «Garant d'une juste cause aux méthodes manichéennes » («Alger pleure », v. 19).

\section{Médine : défenseur des causes justes}

En choisissant de rompre avec le silence longtemps entretenu autour de ces drames qu'a connus l'Afrique à un moment de son histoire, Médine s'opposerait à la clause d'amnistie des traités de paix, qui dans une logique d'apaisement, impose l'oubli de tous les griefs passés relatifs à la guerre, et interdit de les évoquer. À travers cette évocation, il interpelle la communauté internationale à la repentance, à une reconnaissance des responsabilités d'abord individuelles puis collectives dans les persécutions subies par les victimes et leur environnement. Cette démarche semble dès lors être une voie royale pour la réparation. Cette approche qu'il adopte l'inscrit dans le sillage déjà emprunté par certains politiciens français à l'instar de Christiane Taubira et de l'ex-président Hollande.

La loi Taubira, du nom de l'ex-ministre française de la Justice, Christiane Taubira, est votée le 10 mai 2001 et instaurée le 21 mai 2001 en France. Elle reconnaît la traite négrière et l'esclavage comme crime contre l'humanité et, instigue la reconnaissance du 10 mai comme journée du souvenir de l'esclavage et de son abolition. Des années plus tard, précisément le 29 octobre 2016, au cours d'une cérémonie d'hommage rendu aux Tsiganes, victimes du régime de Vichy durant la Seconde Guerre mondiale, le président François Hollande déclare que : «La République reconnaît la souffrance des nomades qui ont été internés et admet que sa responsabilité est grande dans ce drame. » Loin de percevoir cette reconnaissance comme un simple fait de discours, il faut néanmoins l'appréhender comme une source de motivation pour les victimes, car elles sont en permanence à la quête des ressources, surtout psychologiques pour continuer à vivre avec ce lourd fardeau qui leur est imposé par un passé aux ambitions mal négociées.

31 Médine prend conscience du fait que le contexte historique africain est profondément marqué par de douloureux événements historiques et traumas liés à son passé, qui malheureusement continuent de hanter et de déstabiliser son présent. La démarche artistique d'insérer la mémoire et l'histoire du continent dans sa composition vise à soigner l'oubli mémoriel inhérent aux sociétés africaines actuelles, qui ne maitrisent pas toujours leur histoire. Cette écriture est donc cathartique, puisqu'elle se manifeste par la diffusion de la mémoire de l'histoire en vue de guérir l'ignorance et de 
réconcilier la société contemporaine avec ses origines, tout en libérant le peuple de cette imposture dans laquelle il baigne encore.

\section{Médine, jeunesse africaine et perte de repères}

Cette rupture de la société africaine d'avec son passé a des conséquences sur la jeune génération africaine contemporaine. En effet, la classe juvénile, de manière générale, se caractérise par une perte de repères dont certaines des plus grandes manifestations sont entre autres: l'acculturation, le goût de l'ailleurs et le tout attaché à un dépaysement sans précédent. En lanceur d'alerte, Médine, se fait éclaireur de cette jeunesse qui manifestement, à cause de l'ignorance de son histoire, manque de modèle en vue de la construction d'une Afrique fière et jalouse de ses valeurs. Les qualités qu'incarne Kunta pourraient alors être capitalisées par la jeunesse pour venir à bout des difficultés qu'elle rencontre au quotidien. Bien qu'il soit cerné par ses bourreaux, Kunta se bat pour sa dignité, sa liberté et celles de son peuple. C'est une attitude que l'Afrique devrait adopter, elle qui est en proie à plusieurs maux majoritairement issus de la colonisation qui a bouleversé ses habitudes socioculturelles et politiques, avec à sa tête des dirigeants qui manifestement ne servent pas toujours le bas peuple.

Ce bouleversement se traduit également par la perte des valeurs qui faisaient jadis la fierté du continent. Il y a lieu de se souvenir que la capture de Kunta n'aurait sans doute pas été possible, du moins pas évidente si les jeunes de Djouffouré ne s'étaient pas laissé soudoyer par les esclavagistes. En effet, profitant de leur faiblesse, de leur cupidité, les bourreaux de Kunta vont user de la corruption pour se payer leurs services.

34 À travers ce récital, l'on peut comprendre que Médine va en guerre et condamne cette attitude qui constitue pour l'Afrique, l'un des grands fléaux contribuant à sa stagnation. Le développement de l'Afrique est à ces jours spolié ; la faute à la mal gouvernance qui érige au plus haut sommet des États, des systèmes qui ne favorisent pas son développement, encore moins le bien-être des populations. Cette manière de faire passe de devenir une norme auprès de ceux qui auront la responsabilité de conduire les affaires républicaines. Les mêmes causes produisant régulièrement les mêmes effets, il y a lieu d'être dubitatif quant à la capacité de cette jeunesse, déjà à l'image de certains gouvernants actuels, de donner un visage reluisant au continent, étant donné que les écarts ont été normalisés, au détriment des normes sociales de bonne gouvernance alors écartées. Ce qui justifie cette interpellation non seulement de la jeunesse africaine, mais aussi des gouvernants, qui semblent vouloir léguer à la postérité un héritage malheureusement empoisonné. Très souvent qualifiée de fer de lance du continent, cette jeunesse devrait s'armer de valeurs à la hauteur des attentes placées en elle.

\section{Conclusion}

Au regard de tout ce qui précède, il y a lieu de dire que le choix opéré par Médine de consacrer des titres de son riche répertoire musical à deux périodes déterminantes de l'histoire du continent n'est visiblement pas un fait anodin. Il se donne pour objectifs de pointer du doigt ce qui ne va pas, de dénoncer l'immobilisme ainsi que de faire vivre la vérité historique. Prenant appui sur la théorie postcoloniale, on a pu se rendre 
compte que Médine, résolument tourné vers un style marqué par son engagement pour une cause juste, à savoir la restitution du véritable passé africain, passe par l'évocation de l'histoire de l'esclavage et celle de la colonisation, pour non seulement dénoncer ce dont ont été victimes les Africains, mais également pour interpeller les uns et les autres à reconnaître leur rôle respectif dans la mise sur pied de ces différentes entreprises d'exploitation. Cette reconnaissance dont il est question est par ailleurs la voie royale pour la réparation des torts causés. Loin d'être une formulation destinée aux seuls bourreaux, l'artiste se fait historien, afin de rendre aux Africains ce qui leur a été caché. L'objectif étant de stimuler chez eux l'idée d'évolution collective, au moment où, face aux défis multiformes qui les interpellent, les évolutions singulières doivent être bannies. Comme un éclaireur, Médine fait valoir l'histoire comme une force. Ainsi quand le peuple est en manque de repères, il fait usage des grands événements historiques comme boussole. Tout ce combat qu'il livre est la preuve que, les artistes issus du continent africain, dans une logique cathartique, se penchent sérieusement sur l'Afrique malade en laissant dans leurs œuvres, les traces d'une esthétique de la dénonciation, et surtout de la réparation.

\section{BIBLIOGRAPHIE}

Asante, Molefi, 1998, The Afrocentric idea, Philadelphie, Temple University Press.

Césaire, Aimé, 1955, Discours sur le colonialisme, Paris, Présence Africaine.

Delaveau, Bernard, Mongnet, Claire et Salifou, André, 1989, Décolonisation et problèmes de l'Afrique indépendante. Histoire classes de Terminales, Paris, Edicef.

Diop, Cheick Anta, 1967, Antériorité des civilisations nègres : mythe ou vérité historique ?, Paris, Présence africaine.

Fanon, Frantz, [1952] 1971, Peau noire, masques blancs, Paris Seuil, rééd. Paris, Seuil, coll. « Point/ Essais ».

Fanon, Frantz, [1961] 2002, Les damnées de la terre, Paris, La Découverte.

Haley, Alex, 1976, Roots: The Saga of an American family, Garden City, Doubleday.

Hegel, Friedrich, [1822] 1965, La Raison dans l'histoire, trad. K. Papaioannou, Paris, Éditions 10/18.

Kattan, Emmanuel, 2002, Penser le devoir de mémoire, Paris, PUF, coll. « Question d'éthique ».

Lescarbot, Marc, 1795, Tableau historique des progrès de l'esprit humain, publication posthume.

Mauron, Charles, 1964, Des métaphores obsédantes au mythe personnel : Introduction à la psychocritique, Paris, José Corti.

Mbembe, Achille, 1988, Afriques indociles. Christianisme, pouvoir et État en société postcoloniale, Paris, Karthala.

Mbembe, Achille, 2005, De la postcolonie. Essai sur l'imagination politique dans l'Afrique contemporaine, Paris, Karthala. 
Obenga, Théophile, 2000, Pour une nouvelle histoire, Paris, Présence Africaine.

Office of the United Nations High Commissionner for refugees, 2000, The State of the World's Refugees 2000: Fifty Years of Humanitarian Action, Marc Cutts (dir.), Oxford, Oxford University Press.

Ricœur, Paul, 2000, La mémoire, l'histoire, l'oubli, Paris, Seuil.

Sartre, Jean-Paul, 1948, Qu'est-ce que la littérature ?, Paris, Gallimard.

Thibaudet, Albert, [1939] 2005, Réflexions sur la littérature, Paris, Gallimard.

Valette, Bernard, 1985, Esthétique du roman moderne, Paris, Nathan.

\section{Corpus}

Médine, 2008, « Kunta Kinté, enfant du destin », Arabian Panther, Because Music, [En ligne], https://youtu.be/U9p_lJ47a2Q.

Médine, 2012, « Alger pleure », Made in, Because Music, [En ligne], https://youtu.be/ SUA5IVtWoxg.

\section{NOTES}

1. Médine, « Kunta Kinté, enfant du destin », Arabian Panther, 2008, v. 32.

2. Sefyu, « La légende », 2006.

3. Cette expression tient de la période esclavagiste pour désigner l'homme blanc.

4. Paul Aussaresses est un ancien général de l'armée française dont le nom reste associé à la torture lors de la guerre d'Algérie. En 2000 il reconnait avoir eu recours à la torture lors de ce conflit de libération.

5. Comme interprétation, cette sagesse africaine veut dire que, si d'aventure vous allez rendre visite à quelqu'un avec une intention belliqueuse, il est fort probable que cette dernière vous accueille avec violence en « légitime défense ».

6. Il s'agit des identités algériennes devenues opaques, noyées dans le mélange avec les réalités socioculturelles françaises pendant la colonisation.

7. Nous faisons usage de cette expression pour traduire l'idée de la construction d'une société dont un diagnostic aurait été fait au préalable, pour mieux identifier les problèmes des populations afin de mieux les résoudre pour une bien meilleure évolution de la société.

\section{RÉSUMÉS}

Notre étude vise à montrer comment, dans le processus de restitution du passé africain longtemps occulté, le rap questionne l'histoire afin de relever les zones d'ombre et faire revivre la véritable histoire du continent dans la mémoire collective universelle. L'hypothèse principale 
est qu'à travers les titres «Kunta Kinté, enfant du destin » (2008) et "Alger pleure » (2012), Médine, rappeur franco-algérien, rend à l'Afrique les épisodes dissimulés de son passé. L'étude prend appui sur la théorie postcoloniale telle que postulée par Frantz Fanon avec le concept de conscience nationale. Elle aboutit aux résultats suivants: à partir de la thématique de l'impérialisme, de la colonisation, de la guerre, de l'identité, Médine dévoile les atrocités dont ont été victimes les Africains lors de l'esclavage et de la colonisation. En outre, il se fait lanceur d'alerte à l'endroit de la communauté internationale pour une reconnaissance des torts causés, aux fins de réparation. Enfin, en éveilleur de conscience, il interpelle les Africains sur les défis existentiels liés aux conditions d'un développement psychosomatique.

The aim of this article is to analyze how African history is reflected in rap music. Building on Frantz Fanon's concept of national consciousness, this paper argues that the French-Algerian rap musician Medine explores some hidden episodes of the African past through his songs "Kunta Kinte" (2008) and "Alger pleure" (2012). By doing so, Medine points out the atrocities which Africans underwent during the slavery and the colonial periods and he appeals to the international community to take action in order to restore the lost dignity of Africans. Finally, he calls on the Africans to stand up and face new challenges for their development.

\section{INDEX}

Mots-clés : rap, Médine, mémoire, postcolonialisme, impérialisme

Keywords : rap, Médine, memory, postcolonialism, imperialism

\section{AUTEUR}

\section{JULIEN MOWANG NGOULA}

Université de Yaoundé I 